Histoire de l'éducation

122 | 2009

L'enseignement supérieur

\title{
LAOT (Françoise F.), LESCURE (Emmanuel de) (dir.), Pour une histoire de la formation
}

Paris : L'Harmattan, 2008, 135 p.

Pierre Benoist

\section{OpenEdition}

\section{Journals}

Édition électronique

URL : https://journals.openedition.org/histoire-education/1958

DOI : 10.4000/histoire-education.1958

ISSN : 2102-5452

Éditeur

ENS Éditions

Édition imprimée

Date de publication : 1 avril 2009

Pagination : 128-132

ISBN : 978-2-7342-1151-8

ISSN : 0221-6280

Référence électronique

Pierre Benoist, "LAOT (Françoise F.), LESCURE (Emmanuel de) (dir.), Pour une histoire de la formation», Histoire de l'éducation [En ligne], 122 | 2009, mis en ligne le 27 octobre 2009, consulté le 20 mai 2021. URL : http://journals.openedition.org/histoire-education/1958; DOI : https://doi.org/10.4000/histoireeducation. 1958

Ce document a été généré automatiquement le 20 mai 2021.

(c) Tous droits réservés 


\section{LAOT (Françoise F.), LESCURE (Emmanuel de) (dir.), Pour une histoire de la formation}

Paris : L'Harmattan, 2008, 135 p.

\section{Pierre Benoist}

\section{RÉFÉRENCE}

LAOT (Françoise F.), LESCURE (Emmanuel de) (dir.), Pour une histoire de la formation, Paris : L'Harmattan, 2008, 135 p.

1 Ce petit livre rassemble sept articles, dont certains sont issus des contributions au colloque organisé par le Groupe d'étude - histoire de la formation des adultes (GEHFA) à Lyon, le 14 avril 2006, dans le cadre de la huitième Biennale de l'éducation et de la formation, d'autres étant des contributions libres de leurs auteurs.

2 La présentation du volume fait état d'un essor de l'histoire de la formation des adultes, particulièrement sensible dans la deuxième moitié des années 1990. Elle se réfère à une conception très large de l'éducation-formation post-scolaire: générale, culturelle, sociale et professionnelle.

3 Le premier article, «La Ligue de l'enseignement et la réforme des cours d'adultes au tournant du XXesiècle ", est dû à Jean-Paul Martin. Développés par les instituteurs sans rétribution - à l'instigation de Guizot et de Duruy dans un but de " réparation », de perfectionnement ou même de distraction, les cours d'adultes ont à leur apogée, en 1869, touché 800000 personnes, avant de connaître un certain essoufflement. La Ligue de l'enseignement s'y est impliquée surtout dans les années 1890, en s'intéressant principalement aux jeunes sortis de l'école et non encore appelés au service militaire. Au terme de longues discussions, sa doctrine s'est orientée vers la nécessité de créer un enseignement professionnel obligatoire pour les jeunes et pour les employeurs, même si les réalisations de la loi du 2 août 1918 sur l'enseignement agricole et de celle du 
25 juillet 1919, dite « loi Astier ", qui a créé les cours professionnels, n'ont pas été à la hauteur des attentes. La relance des cours d'adultes des années 1890 n'aurait donc été qu'une période transitoire avant l'apparition d'une demande de professionnalisation.

4 L'article d'Antoine Prost, "Jalons pour une histoire de la formation des adultes 1920-1980 »,distingue trois périodes. La première se situe avant 1940. L'ensemble " périscolaire » est alors composé de mouvements d'origine catholique (qui ont après la guerre essaimé vers des organisations syndicales ou des clubs : Confédération française démocratique du travail, Cercle national des jeunes agriculteurs, Citoyens 60), et d'organismes laïques dans la mouvance de la Ligue de l'enseignement (Union française des œuvres de vacances laïques, Union françaises des œuvres laïques d'éducation physique), auxquels se sont joints les Centres d'entraînement aux méthodes d'éducation active et les Auberges de jeunesse. L'ensemble formant «l'éducation populaire " remonte aux "centres sociaux » de la fin du XIXe siècle, d'inspiration confessionnelle, qui visaient à la «moralisation" de la classe ouvrière, et aux universités populaires, d'inspiration syndicale et socialiste. Quant à la «formation professionnelle», elle remonte aux compagnonnages, aux sociétés philanthropiques, passe, entre les deux guerres, par des actions de perfectionnement professionnel pour les cadres (Conservatoire national des arts et métiers, Commission d'étude générale d'organisation scientifique, Chambres de commerce) et, dans les années 1930, par des opérations de formation à destination des chômeurs, notamment à l'initiative de l'Union des industries et des métiers de la métallurgie.

5 Une seconde période voit se dérouler des tentatives de contrôle du secteur par l'Éducation nationale quifont suite à la période de Vichy. L'État français a créé une administration de la Jeunesse et une administration des Sports qui ont été rattachées à l'Éducation nationale à la Libération, avant de lui être ôtées par la Ve République. Dans le domaine de la formation professionnelle, l'Éducation nationale a hérité du régime de Vichy les centres de formation professionnelle, qui sont devenus des centres de formation d'apprentis, et qui appliquaient la méthode de formation de l'ingénieur suisse Carrard. Ces centres sont demeurés dans son giron et ont suivi l'évolution du système scolaire. Mais l'Éducation nationale a échoué à contrôler l'éducation populaire, largement investie par les mouvements associatifs, notamment par Peuple et culture. Elle a lancé en 1956 une offensive dans ce domaine, avec le concours de la Ligue de l'enseignement, mais la tentative a échoué par suite de la réticence des mouvements associatifs, de l'hostilité des catholiques et des difficultés de financement.

Dans la troisième période, l'Éducation nationale se voit réduite au domaine scolaire dans un contexte de développement des attributions à des ministères concurrents (Culture, Travail, Jeunesse et Sports). L'animation socio-culturelle, confiée aux centres sociaux des grands ensembles, les maisons de jeunes et de la culture, les institutions d'éducation surveillée, se développent hors de son influence. La promotion sociale est instituée sous un régime interministériel, la formation dans les entreprises se développe dans les années 1950 et 1960, et la loi du 16 juillet 1971 ne laisse à l'Éducation nationale qu'un rôle modeste de prestataire de service.

7 Dans cet exercice panoramique et diachronique, A. Prost a pris comme fil conducteur la place de l'Éducation nationale, en sous-estimant parfois le rôle qu'elle a tenu, par exemple dans les actions de la Promotion du travail à partir de 1948 (qui avaient au moins autant d'importance que la Promotion sociale qui lui a succédé, en empruntant 
d'ailleurs ses moyens), ou celui qu'elle joue encore dans le dispositif de certification, dont elle inspire la méthodologie. Il resterait aussi à faire la part, dans son échec relatif, de ce qui est inhérent à des défauts constitutifs et de ce qui est imputable à des animosités idéologiques et à des intérêts économiques.

8 Jean-Marc Huguet, dans "Inscription pédagogique et permanence éducative ", s'attache à la formation de trente mille ouvriers et techniciens d'EDF en un demi-siècle, conformément aux statuts de l'entreprise. L'origine de l'opération remonte au régime de l'État français. Un document daté de 1940 énonce les objectifs officiels de l'opération et ses principales applications. L'auteur invite à réfléchir aux évolutions du sens donné à ce texte au cours des années, et notamment à l'époque actuelle.

9 Dans « Un film comme source pour l'histoire : promotion sociale et rapport au savoir, le rôle méritoire des épouses », Françoise F. Laot s'intéresse, à partir du film « Retour à l'école? », produit par le Centre universitaire de coopération économique et sociale (CUCES) et l'Office de radiotélévision française en 1966, aux témoignages qu'il apporte sur les adultes en formation et leur environnement social, et notamment sur le rôle des épouses. C'est aussi l'occasion de revenir sur l'histoire du CUCES, sur la réforme des cours du soir que Bertrand Schwartz y a menée, et de décrire l'origine de la notion de « rapport au savoir » dans ce Centre et ses développements, le film se prêtant à l'étude de cette notion dans un groupe de personnes en formation.

Le statut des formateurs d'adultes et leur formation sont l'objet de l'article d'Emmanuel de Lescure: "Les formateurs d'adultes et leur professionnalisation: du rejet à la fascination. Un exercice de rétrodiction (1960-2000)». Dans les années 1960, il était généralement admis que la formation des adultes devait être donnée "par démultiplication" par des agents en service, sans création d'une catégorie professionnelle nouvelle. C'était en particulier la position de la Confédération française démocratique du travail et de Force ouvrière; la Confédération générale du travail demandait au contraire l'intervention de l'Éducation nationale et de l'Association pour la formation professionnelle des adultes. L'unanimité se faisait sur la nécessité de former des formateurs de formateurs. L'auteur expose comment les opinions se sont progressivement modifiées pour aboutir à une convention collective nationale des organismes de formation en 1988, l'élément déterminant ayant été le besoin de formateurs pour l'encadrement des stages de jeunes dans les années 1980.

11 « De l'éducation permanente à l'éducation et la formation tout au long de la vie »est le titre de l'article de Noël Terrot, ancien directeur du Centre universitaire d'information, de documentation et de recherche sur l'éducation permanente (CUIDEP) de l'Université Pierre-Mendès-France de Grenoble. Après avoir rappelé les trois composantes de l'éducation des adultes: éducation ouvrière, éducation populaire, formation professionnelle, et noté les caractéristiques françaises en matière de formation d'adultes (recours au congé de formation et ambiguïté de la relation entre le mouvement ouvrier et la mouvance intellectuelle), l'auteur s'attache à l'émergence du concept d'éducation et de formation tout au long de la vie, qu'il situe dans les instances de la Communauté européenne, l'année 1996 étant proclamée par la commissaire Edith Cresson « année européenne de l'éducation et de la formation tout au long de la vie ». $\mathrm{N}$. Terrot s'interroge ensuite sur les exigences que comportent les différents objectifs assignés à ces termes et croit discerner des éléments favorables à la concrétisation de ce concept: demande de personnel adaptable, désir de se former pour progresser, 
révélé par la demande de validation des acquis de l'expérience, permanence de l'exigence républicaine d'égalité sociale.

12 À la différence des auteurs de la présentation du volume, Guy Brucy regrette, dans «Plaidoyer pour une socio-histoire de la formation », le faible intérêt pour l'histoire de la formation des adultes, qu'on peut expliquer par l'apparent consensus social qui règne sur le sujet. Une socio-histoire de la formation aurait pour objectif de problématiser la notion, en montrant que les orientations qui se sont imposées n'étaient pas fatales, qu'elles sont le résultat d'affrontements. G. Brucy cite en exemple la loi du 16 juillet 1971, qui est présentée comme innovante alors que la plupart de ses dispositions préexistaient, tel le principe du conventionnement (il aurait pu ajouter que l'essentiel du dispositif attaché à cette loi, à l'exception de l'obligation du financement, est intégralement repris de la loi de 1966 conçue par Michel Debré). En inscrivant la formation dans le droit du travail, les auteurs de la loi ont privilégié le salarié par rapport au citoyen; en légitimant l'entreprise comme lieu de formation, ils déqualifient l'Éducation nationale, ce qui rejoint les évolutions mentionnées par A. Prost dans le même ouvrage.

13 Parmi les chantiers possibles, à mener dans l'interdisciplinarité, G. Brucy retient l'interrogation des concepts et des mots, l'étude des variations de sens des vocables, la biographie des personnes qui ont impulsé les évolutions, la remise en question des dates qui servent de repères chronologiques, l'étude des pratiques de formation à travers les archives des entreprises.

Ce petit ouvrage montre la diversité des sujets que peut suggérer l'expression « histoire de la formation ». Aux orientations énoncées par G. Brucy, il y aurait peut-être lieu d'ajouter une définition plus précise du champ de ce qu'on entend sous le terme de formation. Dans l'ouvrage étudié, la formation s'entend implicitement comme formation des adultes ou, en tout cas, des personnes non situées dans le champ scolaire ou universitaire. Ne faudrait-il pas trouver un vocable adapté pour délimiter ce champ? Mais en même temps, il faudrait aussi éviter la constitution, par la vertu des mots, deux univers qui s'ignorent ou s'excluent. Un chantier de plus? 